\title{
Innovation and Advantage of the DICOM ECG Standard for Viewing, Interchange and Permanent Archiving of the Diagnostic Electrocardiogram
}

\author{
T Hilbel $^{1,2}$, BD Brown $^{3}, \mathrm{~J}_{\text {de Bie }}{ }^{4}, \mathrm{RL}_{\text {Lux }}{ }^{5}$, HA Katus $^{1}$ \\ ${ }^{1}$ Division of Cardiology, University of Heidelberg, Heidelberg, Germany \\ ${ }^{2}$ University of Applied Sciences, Gelsenkirchen, Germany \\ ${ }^{3}$ Mortara Instrument, Inc, Milwaukee, WI, USA \\ ${ }^{4}$ Mortara Rangoni Europe srl, Casalecchio di Reno, Italy \\ ${ }^{5}$ University of Utah, CVRTI, Salt Lake City, UT, USA
}

\begin{abstract}
The DICOM standard is a set of rules that allows medical images to be digitally exchanged, viewed and archived. Since the year 2000 the widely used DICOM standard has included rules for diagnostic ECG waveforms, but for a long time, no ECG manufacturer had marketed electrocardiographs that support the DICOM waveform standard. It was spring 2006 before the first ECG manufacturer announced its adoption of the DICOM standard for diagnostic electrocardiographs. Diagnostic 12 lead DICOM ECGs sent to DICOM network servers can be reviewed on DICOM workstations in conjunction with typical DICOM images like cardiovascular angiographic $x$-ray and cardiac US images. In DICOM the clinician has access to comprehensive medical information that provides essential decision support for evaluating patients. The implementation of a vendor neutral DICOM solution allows healthcare providers to connect a variety of electrocardiographs from different vendors to DICOM servers and no proprietary ECG management system for ECG data will be required.
\end{abstract}

\section{Introduction}

The DICOM standard is a set of rules that allows medical images to be exchanged between image acquisition systems (e.g. x-ray, ultrasound, nuclear cardiology, CT, MRI), image archival systems, and image workstations within hospitals. The widely used DICOM standard [1] also includes rules for diagnostic ECG waveforms. The vendor neutral standard was defined in the year 2000. But for a long time, no ECG manufacturer had marketed electrocardiographs that support the DICOM waveform standard. It was spring
2006 before the first ECG manufacturer announced its adoption of the DICOM standard for diagnostic electrocardiographs. The electrocardiographs interface can connect to DICOM image archive servers. DICOM servers nowadays are available in most hospital environments. So any stored DICOM ECG can be shared and retrieved from hospital DICOM servers. DICOM is a mature, widely deployed communications interface to medical image acquisition modalities. Besides data storage, DICOM also supports reporting and workflow events like procedure scheduling. DICOM is always a vendor neutral implementation. This article focuses on the advantage of using the ECG DICOM standard for the hospital IT infrastructure and for the clinicians.

\section{Methods}

The electrocardiographs ELI 250 and ELI 350 (both Mortara Instrument, Inc) can come with a DICOM compatible interface. Besides DICOM ECG waveform storage, the interface supports the DICOM modality worklist (MWL).

\section{Results}

Any diagnostic 12 lead DICOM ECG tracing can be reviewed on DICOM workstations in conjunction with typical DICOM images like cardiovascular angiographic x-ray, cardiac US, cardiac MRI and nuclear cardiology images (Figure 1). The clinician has access to comprehensive medical information that provides essential decision support for evaluating patients. DICOM is uniquely able to unite diagnostic images with the diagnostic waveform. The DICOM ECG format supports the further integration of clinical data for patient-oriented electronic health record systems. Because the ECG is stored as a full fidelity waveform 


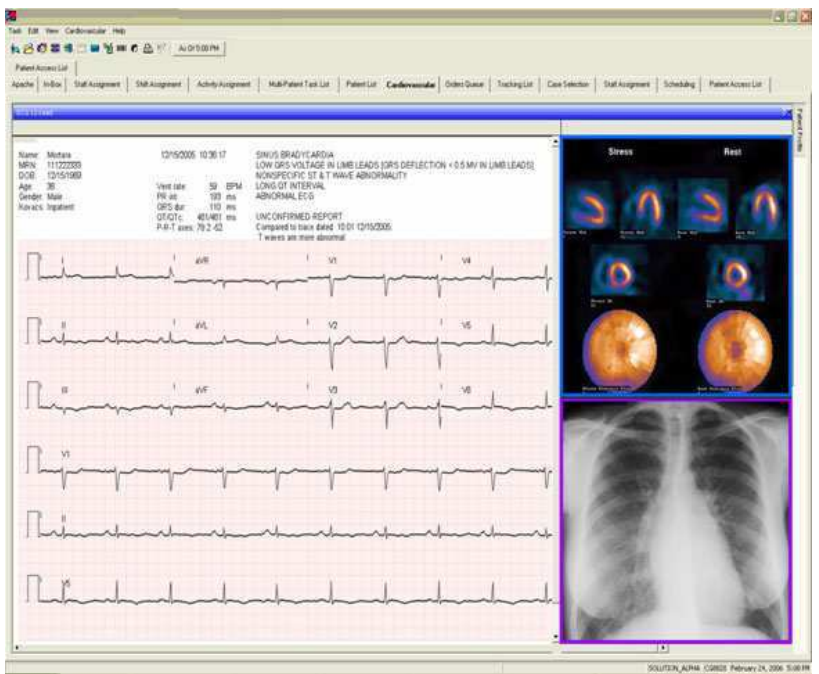

Figure 1. DICOM is able to unite diagnostic images with diagnostic ECG waveforms. The clinician has access to comprehensive medical information that provides essential decision support for evaluating patients.

and not as a static image like a PDF file, it is possible to measure with calipers, make a superposition, or display median beats and do interpretation and ECG over reading within the DICOM workstation (Figure 2). Findings and measurements stored in the annotation section of the waveform object can be queried from the DICOM archive. So it is possible to search hospital wide for all patients with the ECG diagnosis "anteriolateral infarction", for example. And the results can be combined with findings like an occlusion of the coronary artery that are reported in DICOM structure report (DICOM SR) fields of cardiovascular angiographic x-ray images.

The implementation of a standards-based DICOM solution allows healthcare providers to connect a variety of electrocardiographs from different vendors to a single server. For medical facilities there will no longer be a need for a standalone ECG management system with vendor specific ECG review software. In DICOM, ECG data are not proprietary because the digital DICOM ECG file format is an open standard. The files can be accessed by all software products that support the display of diagnostic 12 lead DICOM ECG waveforms. DICOM has full support for ECG and allows hospitals to consolidate information systems. DICOM allows the collection of data from multiple care areas and from different modalities into a single clinical database that is accessible across the whole hospital IT enterprise. Currently without DICOM, the ECG data management system is a proprietary subsystem in the complex hospital IT infrastructure. And so the healthcare providers are locked into purchasing specialized ECG data management systems and electrocardiograph modalities from the same manufacturer. But with the DICOM standard, ECG modalities fit perfectly into an existing multi-modality DICOM hospital IT infrastructure (Figure 3, 4). The use of DICOM as a standard for medical devices can reduce the dependence on custom interfaces to disparate data "islands" with non-standard interfaces.

Medical device manufacturers that declare the implementation of DICOM functionality do also provide a public DICOM conformance statement [2]. This enables any third party company to interface modalities, workstations and archives. Also for the DICOM ECG the public DICOM Validation Toolkit (DVTk) software utility can be used to demonstrate and validate its file structure [3]. The advantage of a public validation software tool is that the device manufacturer and the customer can easily test if the digital data conforms to the open standard.

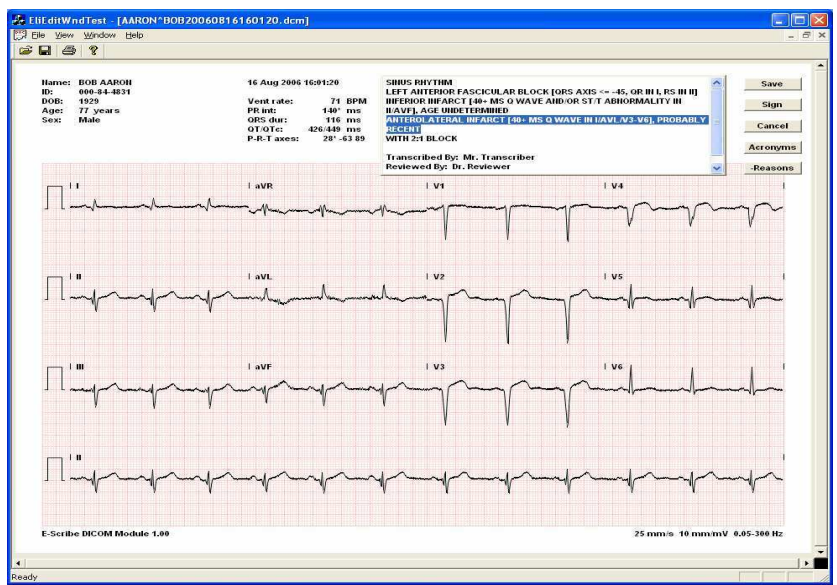

Figure 2. Within a DICOM ECG viewer software application editing ECG interpretation statements for reporting is possible. The measurements are stored in the annotation section of the ECG waveform objects.

For educational purposes ECGs can be transferred to DICOM teaching archives. For purposes of exchange and sharing files can be sent by DICOM email or transferred to any kind of removable digital media ( $\mathrm{CD}$, disc, memory stick etc.). Even the patient could carry his digital ECG with him and exchange it with his medical doctors.

\section{Discussion and conclusions}

Use of the standardized DICOM ECG format will enable the exchange of diagnostic ECG information for patient care, clinical research and for medical ECG training. It has also a value for pharmaceutical research and drug development because researchers can easily exchange and compare their data even if they were acquired with different types of electrocardiographs. 


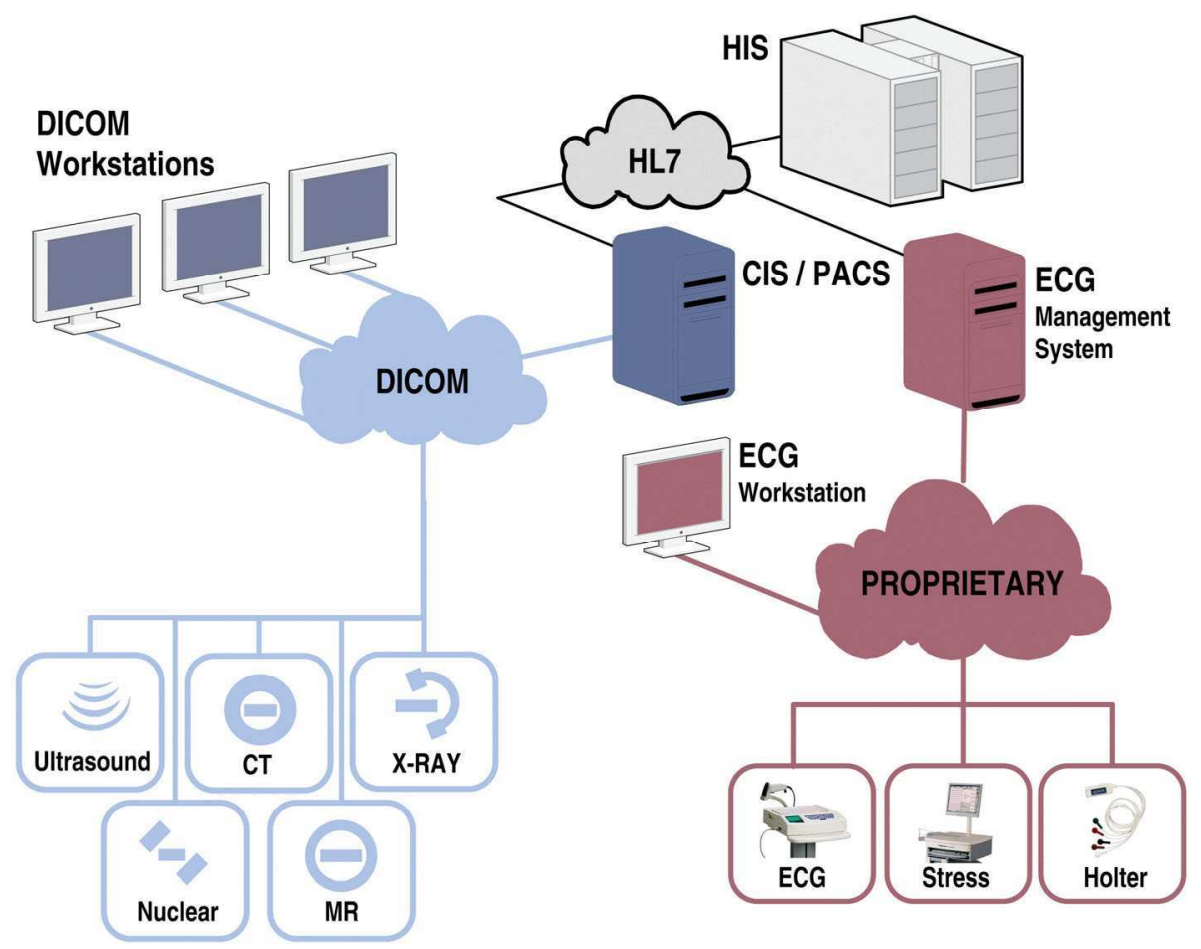

Figure 3. Depicts the current situation of ECG modalities within the hospital IT enterprise environment. The ECG management system is a proprietary subsystem in the complex hospital IT infrastructure. Gateway is a HL-7 server and dedicated ECG viewing clients have to be used.

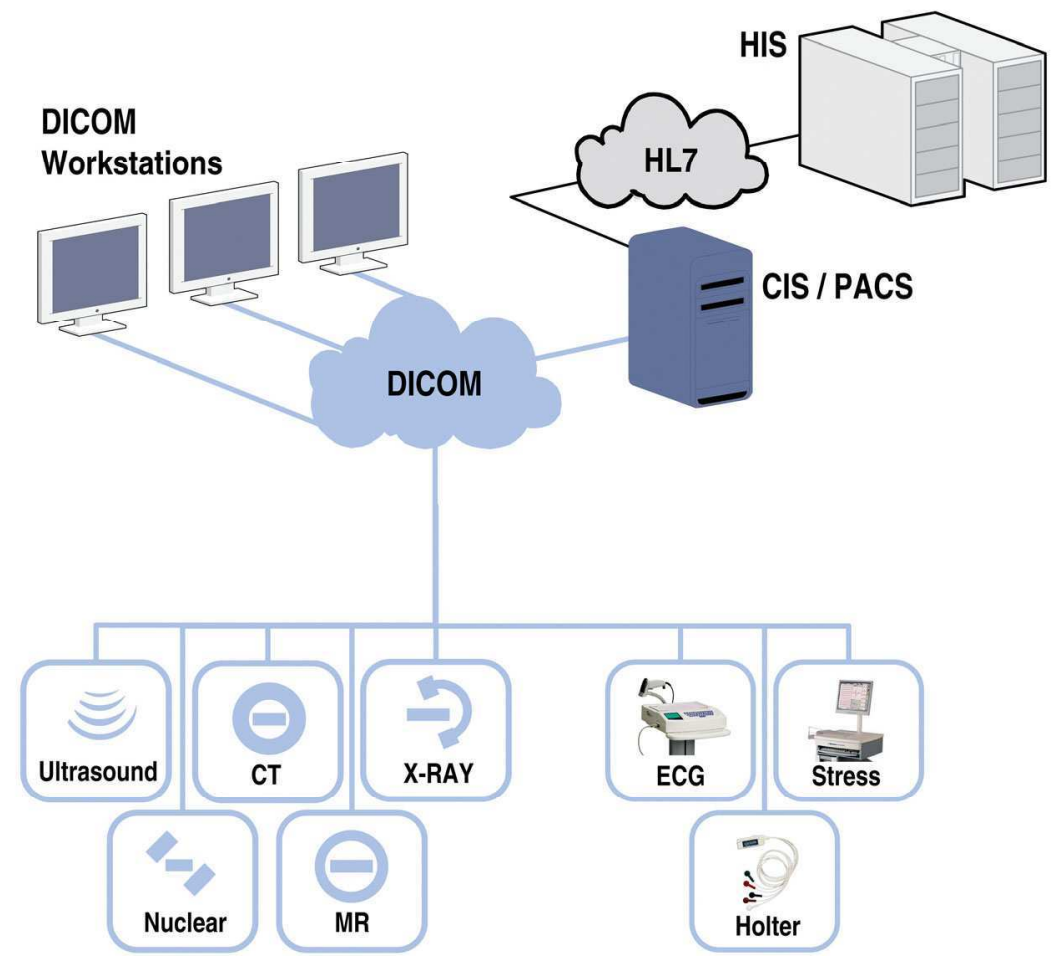

Figure 4. Illustrates the possible advantage of DICOM ECG modalities. The ECG machines fit into the exiting DICOM hospital IT infrastructure. Viewing and reporting can be done on existing DICOM workstations. 
This is an important advantage for experimental and clinical research departments that operate worldwide. But for drug trails the annotated ECG (aECG) remains the preferred standard [4]. In the United States the FDA approves all drugs to be sold and they must know if new drugs will prolong the QT and increase a patient's risk of Torsade de Pointes. So the FDA worked with HL7 to create the aECG standard to meet its drug safety needs. The aECG standard is part of HL7 V3. With this standard the FDA is able to collect and review the ECGs from drug trials. The advantage of aECG is it very general way to represent waveforms and annotations on those waveforms using XML. Annotation examples in aECG are QRS-onset, QT duration, interpretation and location of premature ventricular complexes. Also the aECG includes special clinical drug trial information like the visit, reference event, relative time point and subject treatment group. So the FDA has access to study a new drug's effect on QT before they allow the new drug to be used. But aECG format is not for general healthcare as it is missing important fields like referring physician and the department where the ECG was acquired. Also its HL7 information model is not widely used to communicate with image acquisition modalities.

Before the DICOM ECG waveform standard was published the SCP-ECG digital data format was released as a standard. But SCP-ECG is only for communicating with electrocardiographs over obsolete RS-232 serial connections. SCP-ECG uses complicated compression algorithms that are difficult to implement and test. SCPECG has no support for orders or other workflow. The DICOM standard not only specifies the format of the waveform data and exam reports, but it also specifies how the devices communicate. The DICOM standard supports important workflow events for procedures such as to retrieve requested and scheduled diagnostic ECG exams (MWL = Modality Worklist) or information about the ongoing status of the exam like examination in progress, examination completed or examination canceled (MPPS = Modality Performed Procedure Step). Also the Storage commitment message (SC) informs the acquisition device that the data has been securely archived and can now be deleted. Nowadays all other medical acquisition modalities use DICOM as a more general standard. Therefore the availability of the first commercially distributed DICOM electrocardiographs should encourage other ECG manufacturers to also equip their products with DICOM interfaces. While nowadays DICOM is available for commercial diagnostic resting ECG machines, DICOM capabilities for Stress and Holter ECG modalities will probably be released in the near future. While the waveform objects are well engineered and support all the important aspects of ECG waveforms, Stress and Holter reports will also be supported by the encapsulated PDF object. The DICOM structure report (DICOM SR) template for stress reports is being finalized by the DICOM standards committee, and Holter DICOM SR may still need to be defined. So the challenge for PACS vendors will be the programming or the licensing of appropriate DICOM ECG viewers within their PACS viewing clients.

\section{References}

[1] National Electrical Manufacturer Association (NEMA), Rossyln, VA. Digital Imaging and Communication in Medicine (DICOM), PS 3 2007. 2007: Parts 1 - 18 . ftp.nema.org/ medical/ dicom/final/.

[2] Mortara Instrument DICOM Conformance Statement ELI 150/250 Electrocardiograph V 1.3x. 2007.

[3] Kemper M, Busbridge R. DVT User guide. PDF document Version 2.1. 2005: 1 - 116.

[4] Health Level Seven. HL7 Version 3 Standard: Regulated Studies - Annotated ECG, Release 1. 2004: www.h17.org/V3.

Address for correspondence

Thomas Hilbel MD

University of Heidelberg, Division of Cardiology

Im Neuenheimer Feld 410

69120 Heidelberg, Germany

Phone: ++4962215639780

E-mail: thomas.hilbel@med.uni-heidelberg.de 